This item was submitted to Loughborough's Research Repository by the author.

Items in Figshare are protected by copyright, with all rights reserved, unless otherwise indicated.

\title{
Parametric pendulum based wave energy converter
}

PLEASE CITE THE PUBLISHED VERSION

https://doi.org/10.1016/j.ymssp.2017.06.026

\section{PUBLISHER}

(c) Elsevier

VERSION

AM (Accepted Manuscript)

\section{PUBLISHER STATEMENT}

This work is made available according to the conditions of the Creative Commons Attribution-NonCommercialNoDerivatives 4.0 International (CC BY-NC-ND 4.0) licence. Full details of this licence are available at: https://creativecommons.org/licenses/by-nc-nd/4.0/

\section{LICENCE}

CC BY-NC-ND 4.0

\section{REPOSITORY RECORD}

Yurchenko, Daniil, and Panagiotis Alevras. 2019. "Parametric Pendulum Based Wave Energy Converter". figshare. https://hdl.handle.net/2134/27518. 


\title{
Parametric pendulum based wave energy converter
}

\author{
Daniil Yurchenko ${ }^{\mathrm{a}}$, Panagiotis Alevras ${ }^{\mathrm{b}}$ \\ ${ }^{a}$ Institute of Mechanical, Process \& Energy Engineering, Heriot-Watt University, \\ Edinburgh, EH14 $4 A S, U K$. \\ ${ }^{b}$ Wolfson School of Mechanical, Electrical $\& 3$ Manufacturing Engineering, Loughborough \\ University, Loughborough, LE11 3TU, UK.
}

\begin{abstract}
The paper investigates the dynamics of a novel wave energy converter based on the parametrically excited pendulum. The herein developed concept of the parametric pendulum allows reducing the influence of the gravity force thereby significantly improving the device performance at a regular sea state, which could not be achieved in the earlier proposed original point-absorber design. The suggested design of a wave energy converter achieves a dominant rotational motion without any additional mechanisms, like a gearbox, or any active control involvement. Presented numerical results of deterministic and stochastic modeling clearly reflect the advantage of the proposed design. A set of experimental results confirms the numerical findings and validates the new design of a parametric pendulum based wave energy converter. Power harvesting potential of the novel device is also presented.

Keywords: Wave energy converter, wave energy, parametric pendulum, power take-off, reduced gravity, energy harvesting, Mathieu equation, electromagnetic generator.
\end{abstract}

\section{Introduction}

Energy harvesting has been attracting much attention in recent years, which is reflected in a great number of publications. In some areas, like 
wind energy, there have been significant advances made allowing to pass from a concept up to a commercialization stage, implementing some novel ideas in practice. Wave energy is another promising area the development of which however has been progressing slower than expected due to a number of reasons. Over one hundred fifty various concepts of Wave Energy Converters (WECs) exist and new ones keep appearing quite regularly. Some of the ideas went through the commercialization stage and have been built and deployed, for instance Pelamis, Oyster, OWC, etc [1]. Despite these developments, the cost of wave energy remains significantly high, almost ten times greater than that of coal and almost twice the cost of offshore wind energy [2]. The Carbon Trust identified three major directions where cost reduction can be achieved: device components, operation and maintenance as well as next generation concepts [2]. This becomes especially important in a view of recent bankruptcies of two major players in this sector - Pelamis and Oyster. Thus, it becomes obvious that the race for a new generation of more efficient, inexpensive and robust WECs is still on.

Relatively recently, a novel wave energy power take-off concept has been first proposed in [3]. Its main idea was based on the properties of a parametrically excited pendulum. It is well known that if a pendulum's suspension point is excited harmonically in the vertical direction with a certain frequency, rotational response of the pendulum is possible. The dynamics of such a system can be described by the following nonlinear equation:

$$
\begin{aligned}
& \theta^{\prime \prime}+\gamma \theta^{\prime}+[1+\lambda \cos (\nu \tau)] \sin \theta=0, \\
& \lambda=\frac{A \omega^{2}}{L \Omega^{2}}, \nu=\frac{\omega}{\Omega} .
\end{aligned}
$$

Here $L$ and $\Omega$ are the length and natural frequency of the pendulum, $\gamma$ viscous damping coefficient, $A$ and $\omega$ - the excitation (waves) amplitude and frequency. Thus, using the heaving motion of waves as a parametric exci- 
tation of the pendulum's suspension point it is potentially possible to make the pendulum rotate, which can be used to generate electricity. The major advantage of this elegant idea is that electricity can be generated in a conventional way and would not require any intermediate auxiliary mechanisms. However, the implementation of a parametric pendulum as a power take-off system turned to be more challenging than it seemed. There are three, most important from the authors' point of view, challenges that prevented further development of the concept so far. The first two could be summarized as: how the pendulum would behave in a random sea environment and how to reduce the size of the device. These two challenges are outlined below whereas the third one, concerning the improvement of rotational potential of the pendulum, will be thoroughly addressed in this paper.

The first challenge was to understand whether a sustainable rotational motion could be observed under a sea-like environment excitation, since sea waves could barely resemble a harmonic process. There has been a number of publications related to the rotational potential of a parametrically excited deterministic pendulum, most of which were not concerned with energy harvesting but rather were focused on the deterministic and chaotic response of the pendulum ([4-7] and references therein). Rotational potential is defined as a percentage of rotational motion of the pendulum over the overall time and it directly influences the amount of power generated. Apparently, the average energy of a pendulum in rotations is larger than that of the pendulum in an oscillatory motion, which can be seen from the state-space trajectories of these motions. Later, various combinations of excitations and pendulums have been tested to understand how the direction of the excitation, as well as the interaction of two and more pendulums influence the rotational potential ([8-14] and references therein). Experimental investigations on the 
rotating parametric pendulum and energy extraction can be found in [15-21]. Recently the authors have published a number of papers where a stochastic excitation has been used to model the parametrically excited pendulum behavior under more realistic loading [22-26]. Namely, a harmonic excitation with a mean frequency and random phase modulations has been used to generate a sea like waves with a proper spectrum (Pierson-Moskowitz spectrum was used). It has been found that a sustainable rotational motion of the pendulum can be achieved under a narrow band excitation. Rotational domains with $10 \%$ to over $90 \%$ of rotational motion were identified and it has been shown that the increase of randomness (noise intensity) moves the domains of rotational motion to the region of higher $\lambda$ values, thereby reducing the rotational potential.

The second challenge was related to the physical size of the pendulum. Since the primary parametric resonance occurs when the excitation frequency is twice the natural frequency and an average excitation frequency of waves is about $0.1 \mathrm{~Hz}$ (period of $T=10 \mathrm{~s}$ ), the pendulum's natural frequency should be around $0.05 \mathrm{~Hz}=0.314 \mathrm{rad} / \mathrm{s}$ to bring the pendulum to the primary parametric resonance. Such a frequency is extremely hard to attain for the lumped mass (mathematical) pendulum, since its squared natural frequency is inversely proportional to its length $\Omega^{2}=g / L$ and therefore the pendulum should be around 100 meters long. The size can be significantly reduced if one considers a compound pendulum, which can have a relatively small size and low frequency. The latter can be achieved by a very small radius of gyration, which is impractical since it can be smaller than the diameter of a holding pin and also provide extremely low torque, which is essential for energy harvesting. A reasonable solution to this problem was offered by the authors [12], where dynamics of a pendulum with $N$ arms, rigidly connected 
to a joint hub and equally distributed at intervals of $2 \pi / N$, was investigated (not to be confused with $\mathrm{N}$ pendulums - a system where each pendulum is connected to the end of other). Each arm has a lumped mass, placed at some distance from the hub, so that by changing these distances one can control the pendulum's natural frequency. It has been shown that such an $N$-pendulum system not only can achieve low natural frequency at different distances of the masses from the hub, but also has a number of advantages, offering an opportunity to adjust the pendulum's frequency and moment of inertia (therefore torque) independently.

The third challenge, which is the focus of this paper, concerns the rotational potential of the pendulum, namely how to facilitate rotations of the pendulum under the standard sea state conditions. It has been outlined above, that it is possible to observe a rotational response of the pendulum, however, it is reached at values of $\lambda \geq 1$ for $\gamma=0.3$ and $\lambda \geq 0.5$ for low value of damping $\gamma=0.01$. Rewriting $\lambda=A \omega^{2} / g$, where $g$ is the acceleration of gravity, one can see that for the low damping case $A \omega^{2} \geq g / 2$, which is impossible to satisfy in the regular sea state with a significant wave height of about $1-1,5 \mathrm{~m}$, because $\omega^{2} \approx 0.4 \mathrm{rad} / \mathrm{sec}$ for $T=10 \mathrm{~s}$ or $\omega^{2} \approx 0.8 \mathrm{rad} / \mathrm{sec}$ for $T=7 \mathrm{~s}$ waves. This issue could be overcome by using some mechanism to increase the input frequency, for instance a gearbox. However, the latter are known, from the extensive experience with wind turbines, to be very capricious and not very much reliable, thus would not provide a feasible solution to the problem. In this paper we offer another way out of this predicament.

Since parameter $\lambda=A \omega^{2} / g$ depends purely on the property of the waves, which cannot be changed or controlled, the gravity force is the only component that can be varied. Apparently the acceleration of gravity itself cannot be changed, however, the influence of the gravity force onto the system can. 
That is the main point of the novel WEC design that makes it very practical. Therefore in Secton 2 the new idea of a WEC concept is outlined and a new governing equation of motion is derived. This equation is solved numerically for deterministic and stochastic wave models and presented in the form of parametric space maps in Section 3. In Section 4 new results of the experimental studies are presented for the proposed WEC design.

\section{The low gravity environment WEC concept}

The first concept of a parametric pendulum WEC was proposed as a heaving point absorber (Fig 1), with the pendulum's suspension point moving up and down due to bobbing motion. In this case the gravity force provides the only restoring force for the pendulum:

$$
M l^{2} \ddot{\theta}+b \dot{\theta}+M g l \sin \theta=M l \ddot{f}(t) \sin \theta
$$

where $\theta$ is the angle of inclination, $f(t)$ - excitation force, $l$ - the length of the pendulum or the distance from the suspension point to the lumped mass $M$. If the excitation force is perfectly harmonic, $f(t)=A \cos (\omega t)$, then the previous equation may be rewritten:

$$
\ddot{\theta}+2 \alpha \dot{\theta}+\left[\frac{g}{l}+\frac{A}{l} \omega^{2} \cos (\omega t)\right] \sin \theta=0
$$

When the inclination angle is small and $\sin \theta \approx \theta$ eq. (3) is known as Mathieu equation. Eq. (3) can be written in a different form if a new non-dimensional time $\tau$ is introduced:

$$
\begin{gathered}
\theta^{\prime \prime}+[1+\lambda \cos (\nu \tau)] \sin \theta=0, \\
\tau=\Omega t, \Omega^{2}=\frac{g}{l}, \nu=\frac{\omega}{\Omega}, \lambda=\frac{A}{l} \nu^{2}=\frac{A \omega^{2}}{g}
\end{gathered}
$$

where prime denotes derivative with respect to $\tau$. Thus one arrives to eq. 1, which has an extra damping term that can account for an overall energy 
dissipation mechanism. The previous investigations have shown that for the pendulum's rotational motion it is required to have $A \omega^{2} \geq g$ [22]. Thus, if the influence of the gravity force is reduced, it is perhaps possible to achieve the rotational motion for lower values of $\lambda$, which would make the concept feasible in the regular sea state. This case of the reduced gravity force will be called from now on as the low gravity environment.

To achieve the low gravity environment let's place the pendulum so that the pendulum's plane of rotation and the water surface form an angle $\beta$, then its normal $\vec{n}$ (parallel to the axis of rotation) makes angle $90-\beta$, as shown in Fig 2. Then the in-plane restoring force due to gravity will be $m g \sin \beta$ and therefore the gravity force will be reduced.

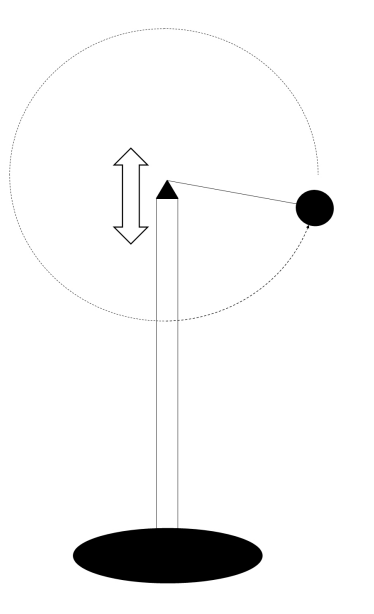

Figure 1: Sketch of the original parametric pendulum WEC concept.

The low gravity case leads to a novel design of the WEC based on the parametric pendulum, schematically drawn in Fig 3. The device becomes an attenuator type, facing the incident waves and comprises a half-submerged, rectangular in the rotational plane, ramp with angle $\beta$, which is rigidly fixed to the sea bed. The buoy (spherical in the figure) moves along the ramp up and down due to the waves motion. The buoy has a sliding platform with a 

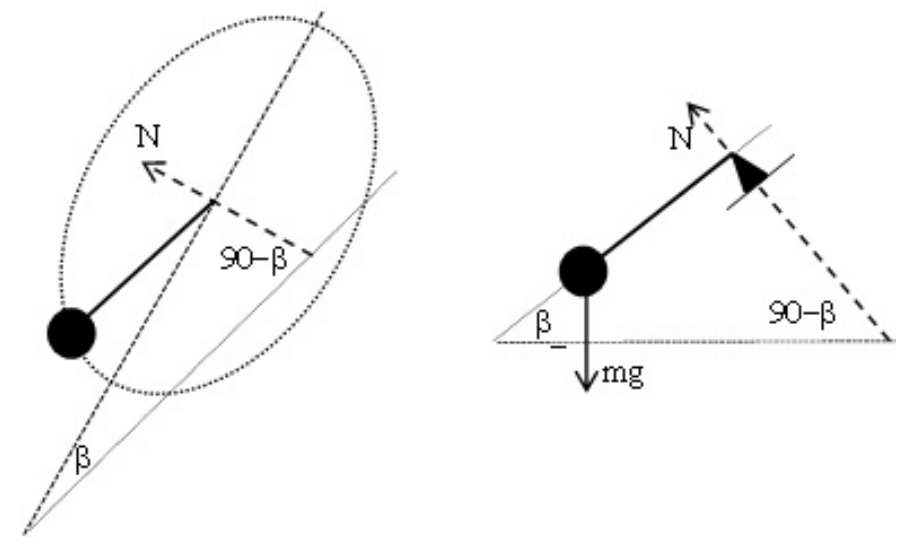

Figure 2: Sketch of the pendulum configuration with reduced gravity force with 3D (left) and side (right) views.

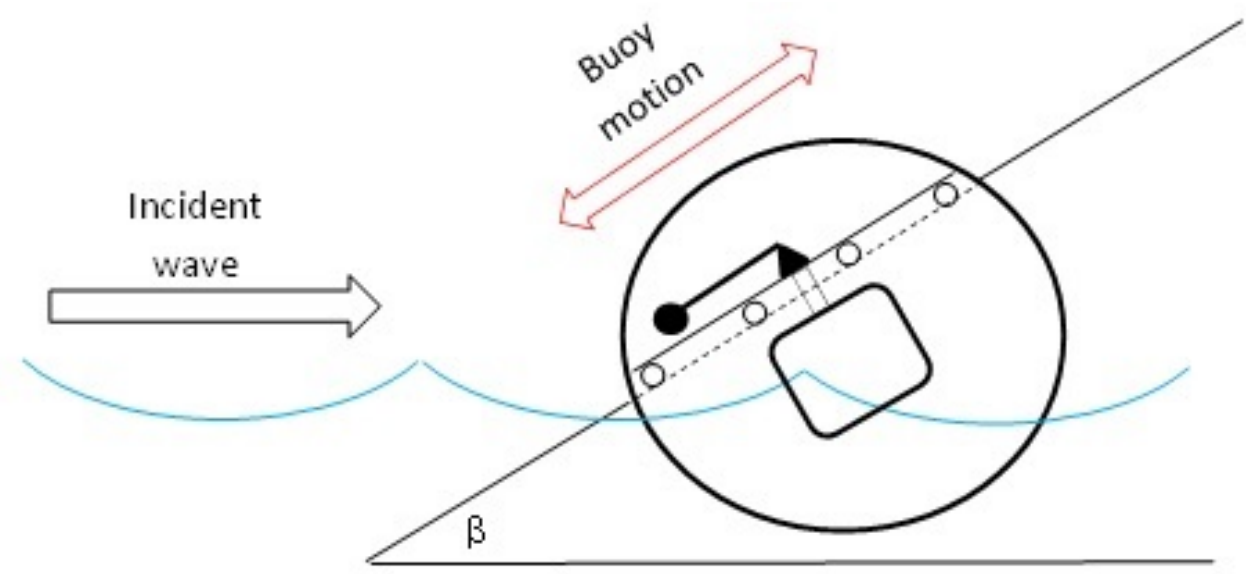

Figure 3: Concept of the parametric pendulum WEC (side view).

$\mathrm{N}$-pendulum system, so that the motion of the buoy provides a parametric excitation to the pendulum's pivot point. The shaft from the pendulum is directly connected to an electrical device which will generate electricity. Using the N-pendulum helps to tune the device to a proper frequency. Moreover the mechanical energy depends on the pendulum mass and its squared distance, thus it has a large inertia that helps to overcome small fluctuations, due to irregular waves, and maintain rotational motion. 
The proposed design allows actively controlling the device through the angle $\beta$, the natural frequency of the pendulum and the amplitude of the excitation. The first parameter $\beta$ plays important role in bringing the dominant rotational domain to low values of $\lambda$, as can be seen later. If the device is rigidly built into the sea bed, angle $\beta$ can be controlled through a hydraulic mechanism. The natural frequency of the N-pendulum can be adjusted by moving masses towards or away from the pivot point. In the case of extremely high waves the excitation amplitude can be limited through the artificial barriers installed in the ramp.

It can be shown that the dynamics of the proposed device for the low gravity case is similar to the one described above with a change in the gravity force:

$$
M l^{2} \ddot{\theta}+b \dot{\theta}+M g l \sin \beta \sin \theta=M l \ddot{f}(t) \sin \theta,
$$

Introducing a harmonic excitation $f(t)=A^{*} \cos (\omega t)$ we write:

$$
M l^{2} \ddot{\theta}+b \dot{\theta}+M g l \sin \beta \sin \theta=-M l \omega^{2} A^{*} \cos \omega t \sin \theta,
$$

where $A^{*}$ is the amplitude along the ramp, so that $A^{*}=A / \sin \beta$. The latter may be thought of as another important advantage of the proposed design - amplification of the excitation amplitude due to the buoy traveling along the inclined ramp. It is interesting that this type of Mathieu equation $(\sin \theta \approx \theta)$ has never been studied before and therefore it is of interest to see what happens to the stability boundaries with changing $\beta$. For that purpose let's rewrite this equation in the form (assuming zero damping and $\omega t=2 s$ )

$$
\begin{aligned}
& \frac{d^{2} \theta}{d s^{2}}+[d+2 e \cos (2 s)] \theta=0, \\
& d=\frac{4 \Omega^{2}}{\omega^{2}} \sin \beta, e=\frac{2 A}{l \sin \beta},
\end{aligned}
$$

which is appropriate for using the well-known expressions for the instability 
boundaries [27] of the first and second instability domains, which are:

$$
\begin{aligned}
& d=1 \pm e-\frac{1}{8} e^{2}, \\
& d=4+\frac{1}{6} e^{2} \pm \frac{1}{4} e^{2} .
\end{aligned}
$$

In Fig 4 instability boundaries of the first and second instability domains, according to (8) are presented respectively. It should be mentioned that the original expressions were valid for small values of $e$, so that higher order terms in $e$ were not accounted for, therefore such a drastically different shape of the first instability domain may be the result of the inadequate representation of this domain for small values of $\beta$ (see the right boundary at low values of $\beta$ ). In general the effect of low gravity is reflected in the shift of the resonant frequencies to the right with simultaneous widening of the domains. The left boundary of the first instability domain is the one that allows the proposed design to succeed since it gets lower with decreasing values of $\beta$. One can also see that it is much harder to achieve the second instability domain at low frequencies than the first one. Thus, in the experimental study, which will be reported later, the pendulum was rotating with the frequency of the excitation, thus we observed rotational motion in the first instability domain.

Let's now get back to our original, nonlinear equation and rewrite it as follows:

$$
\begin{aligned}
& \theta^{\prime \prime}+\gamma \theta^{\prime}+[\sin \beta+\lambda \cos (\nu \tau)] \sin \theta=0, \\
& \lambda=\frac{A^{*} \omega^{2}}{g},
\end{aligned}
$$

It should be noted that the damping ratio $\gamma$ of the physical system will change depending on the moment of inertia of the N-pendulum as well as the angle $\beta$. However, the influence of viscous damping onto the instability of a parametrically excited system is well known from previous numerical studies and therefore is not of major concern here, thus throughout all the simulations this value will be kept constant $\gamma=0.1$. 


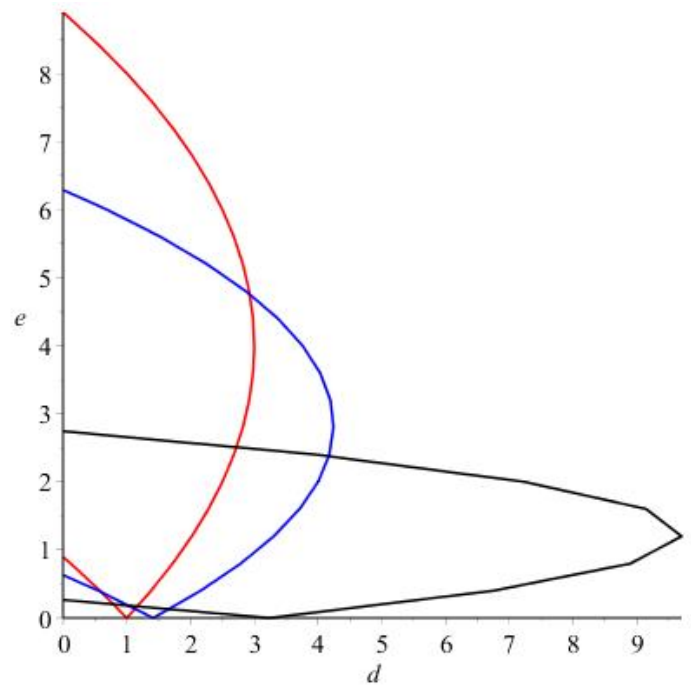

(a)

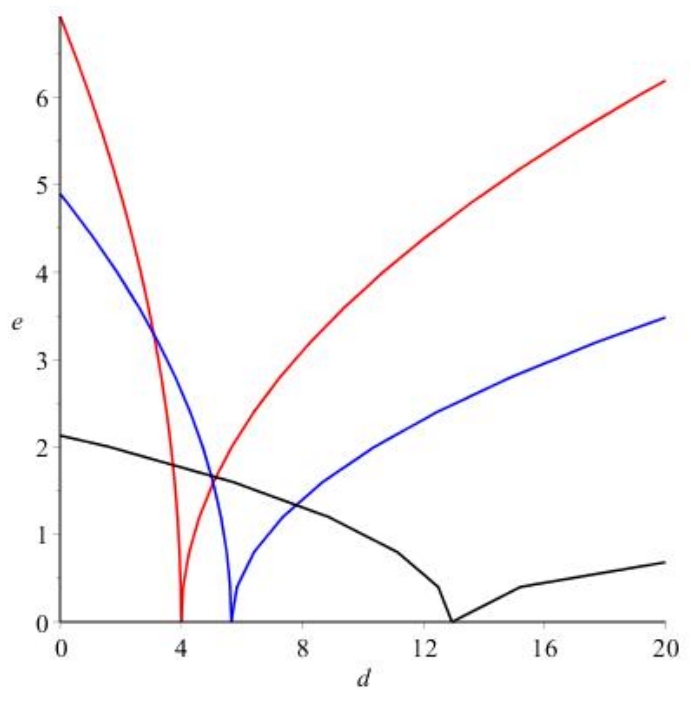

(b)

Figure 4: Instability domains for $\beta=\pi / 2$ (red), $\beta=\pi / 4$ (blue) and $\beta=\pi / 10$ (black); (a) First instability domain; (b) Second instability domain;

\section{Results of Numerical Investigations}

It is of paramount importance to analyze what happens with the rotational potential of the pendulum in the low gravity environment. Numerical modeling remains the major and inexpensive way of investigating performance of WECs [28, 29]. Therefore a set of deterministic and stochastic numerical simulations have been conducted for different values of $\beta$. The results of the deterministic modeling are presented in Figure 5 as parametric space maps (PSMs) of $(\lambda, \nu)$. Red color indicates the regions of dominant rotational motion (over $90 \%$ of time), irrespective of its direction and period, whereas blue color indicates low rotational potential of the pendulum (less than 10\%).

The benefit of the proposed design is elucidated in all the figures since the dominant rotational domains have been shifted downwards to lower values 
of $\lambda$ and to the left, to lower values of $\nu$ thereby increasing the natural frequency of the pendulum. In fact in Figure 5(b) one can observe that one of the two red peninsula went down to values of $\lambda<0.2$. Obviously the traveled distance or the excitation amplitude in the inclined direction $\left(A^{*}\right)$ will be far greater than the height of the wave and therefore angle $\beta$ does not have to be very small. For instance, the rotational domain at $\lambda=0.45$ and $\beta=20^{\circ}$ can be reached for $T=10 \mathrm{~s}$ with $A=1.3 \mathrm{~m}$, which is a very reasonable value for a regular seas state. In fact the annual energy EU distribution along the Atlantic coast indicates the peak value at $T=7-8 \mathrm{sec}$ with $H=2 A=1-1,5 m$, which can be reached by the proposed design with angle $\beta=20^{\circ}$ or lower.

It is of primary interest to see if the rotational motion will be sustained in the random environment. The random phase modulations technique can be used to model the waves excitation with a given mean frequency $\omega$. The former consists of a harmonic process $\lambda \sin (q(t))$, with $q^{\prime}=\nu+\sigma \xi(t)$, where $\nu$ is the mean excitation frequency and $\sigma^{2}=D$ is the noise intensity, so that $\xi(t)$ is Gaussian zero mean noise of unit intensity. An exact analytical expression for the Power Spectral Density function of this process can be obtained by the measuring filters approach [30] and is known as:

$$
S(\omega)=\frac{\lambda^{2} D\left(\omega^{2}+\nu^{2}+D^{2} / 4\right)}{\left.2\left\{(\omega+\nu)^{2}+D^{2} / 4\right\}\left\{(\omega-\nu)^{2}+D^{2} / 4\right)\right\}}
$$

Therefore it is possible to adjust the values of $\nu$ and $D$ to match more or less a given spectrum. This has been done in [31] for the well known PiersonMoskowitz spectrum, and it was found that $D=0.3$. To get more accurate approximation of the PM spectra one can adapt a higher order filter [32]. Thus, the following stochastic model of the parametric pendulum is studied next: 


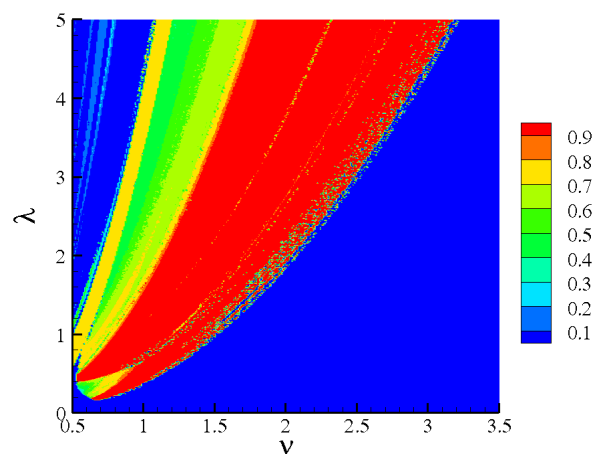

(a)

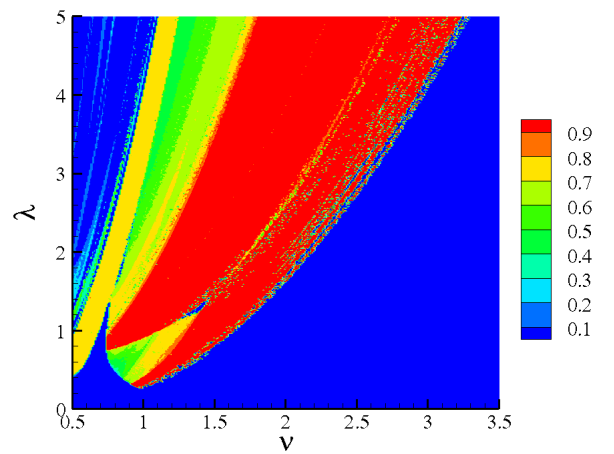

(c)

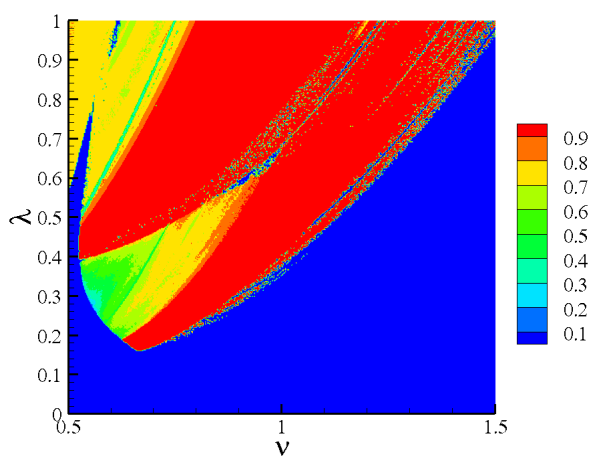

(b)

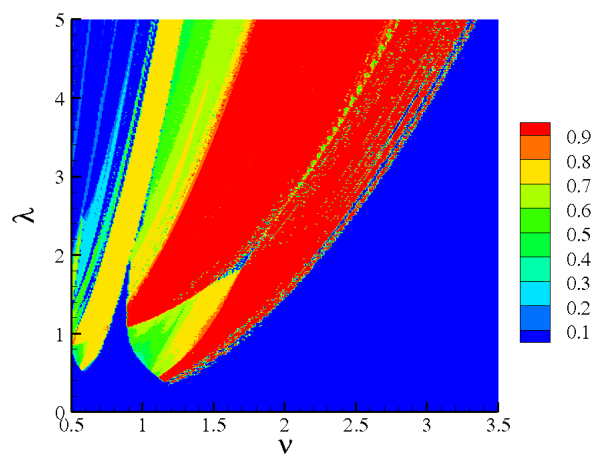

(d)

Figure 5: Rotational potential of a pendulum in low gravity environment for (a) $\beta=10^{\circ}$; (b) Zoom-in case of $\beta=10^{\circ}$ (c) $\beta=20^{\circ}$; (d) $\beta=30^{\circ}$

$$
\begin{aligned}
& \theta^{\prime \prime}+\gamma \theta^{\prime}+[\sin \beta+\lambda \cos q(t)] \sin \theta=0, \\
& q^{\prime}=\nu+\sigma \xi(t),
\end{aligned}
$$

Monte-Carlo simulations based on random number generation are conducted to obtain parameter space maps that depict the regions of rotational motion. Each point is analysed for 300,000 sample periods. The reader is referred to previous work for details of the computations [22].

Two different values of noise intesity $D=0.1$ and $D=0.3$ were selected 
for the investigation with three different values of angle $\beta$, which are demonstrated in Figure 6. The left column corresponds to $D=0.1$ and angles of $\beta=5^{\circ}, 20^{\circ}, 35^{\circ}$ respectively. One can observe here that the red domains have been shifted to the upper right corner, reducing the rotational potential of the pendulum. This tendency is supported by the set of plots in the right column, where there are no dominant rotational domains present at all. Despite that it is important to stress that the lowest rotational boundary has been practically unchanged with the increase of noise, which indicates that $40 \%$ to $70 \%$ of the rotational motion can still be achieved in the regular sea environment. Although these numbers are far from the optimal value, it demonstrates that reasonable amounts of energy can potentially be transformed into electricity, taking into account that the waves power density on average is more than 15 times greater than that of wind, even though the wind turbines averaged annual output is around $30 \%-40 \%$.

\section{Experimental Investigation}

To verify the results of the deterministic numerical modeling a set of experiments was conducted. The experimental rig consists of two mechanical systems: a tri-pendulum and electrical motor, shown in Figure 7. To accommodate the tri-pendulum the frame of $4 m \times 2 m$ with $1 m$ in depth was built. To minimize the motor load, the crank had a counterweight at its free end while the other end had 4 holes at $0.35,0.4,0.45$ and $0.5 \mathrm{~m}$ to allow various excitation amplitudes through the connecting rod (Figure 8(a)). The latter provides a reciprocating motion of the pendulum's suspension point. To avoid the adverse effect of the reciprocating motion, reflected in the presence of higher order harmonics in the excitation, the crank length was selected long enough to render this effect negligible, thereby keeping a single har- 


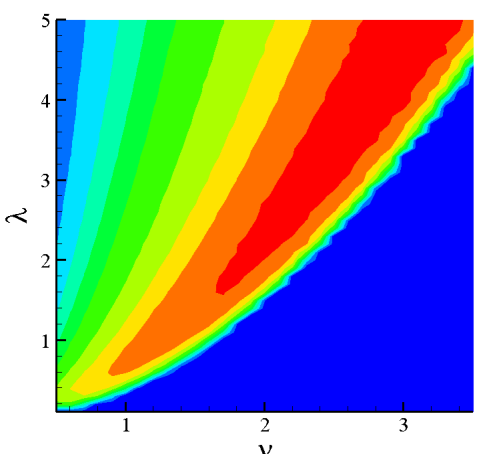

(a)

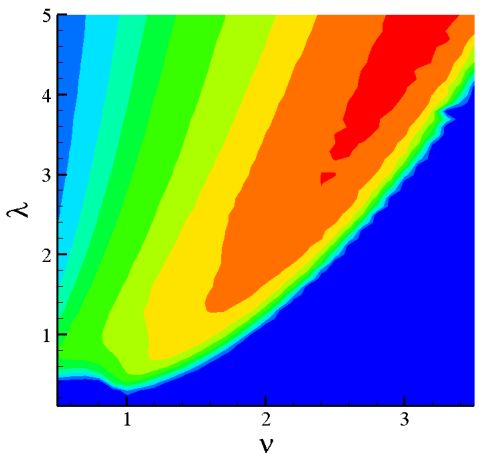

(c)

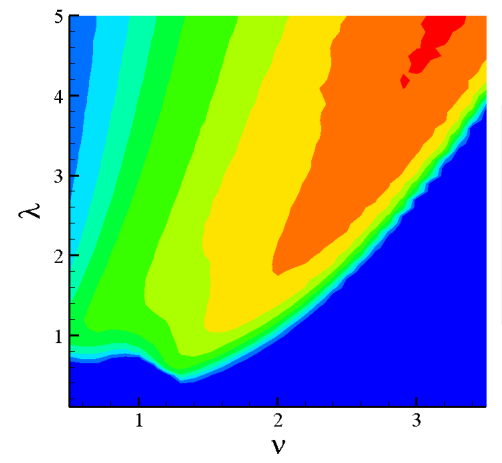

(e)

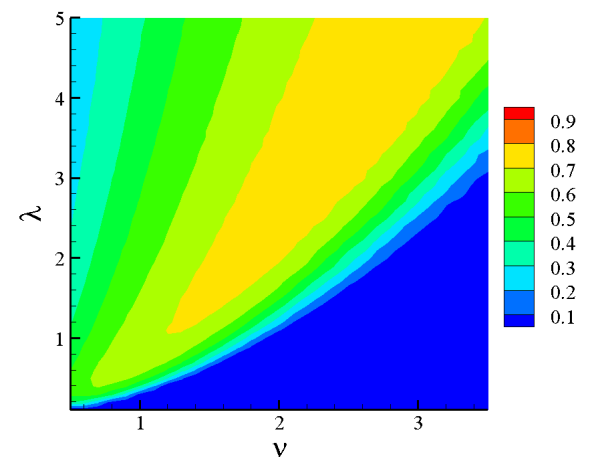

(b)

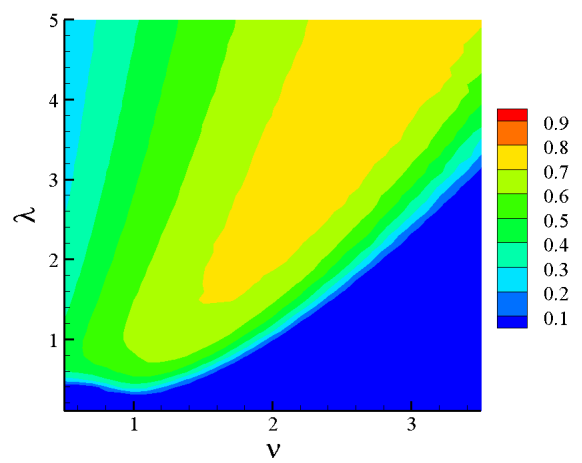

(d)

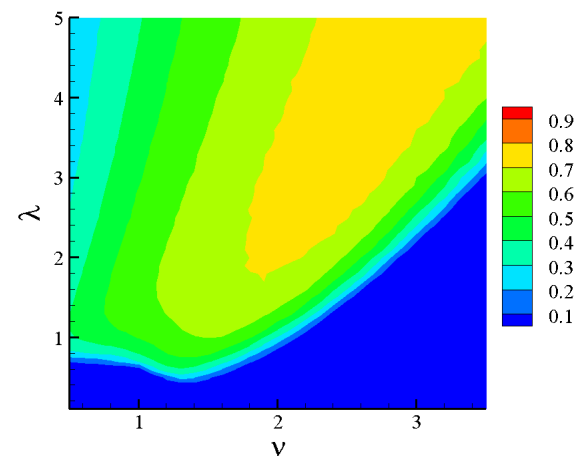

(f)

Figure 6: Stochastic rotational potential of a pendulum in the low gravity environment with $\sigma^{2}=0.1$ (left) and $\sigma^{2}=0.3$ (right) and (a), (b) $\beta=5^{\circ}$; (c), (d) $\beta=20^{\circ}$; (e), (f) $\beta=35^{\circ}$ 
monic in the excitation. The other end of the connecting rod was attached to the tri-pendulum's hub, comprising a sliding mechanism with rollers to move along two rails directing the hub motion. The rig was placed at angle $\beta$ with respect to the floor, as is depicted in Figure $8(\mathrm{~b})$, to simulate the low gravity environment. The tri-pendulum hub at one side was connected to three arms with equal masses, whereas the other side was connected to an angular encoder with resolution of 3000 counts per revolution, providing a digital signal of the tri-pendulum's motion. The signal was analyzed and recorder by a DAQ card managed by bespoke LabView software. More technical detail on the experimental rig can be found in [33].

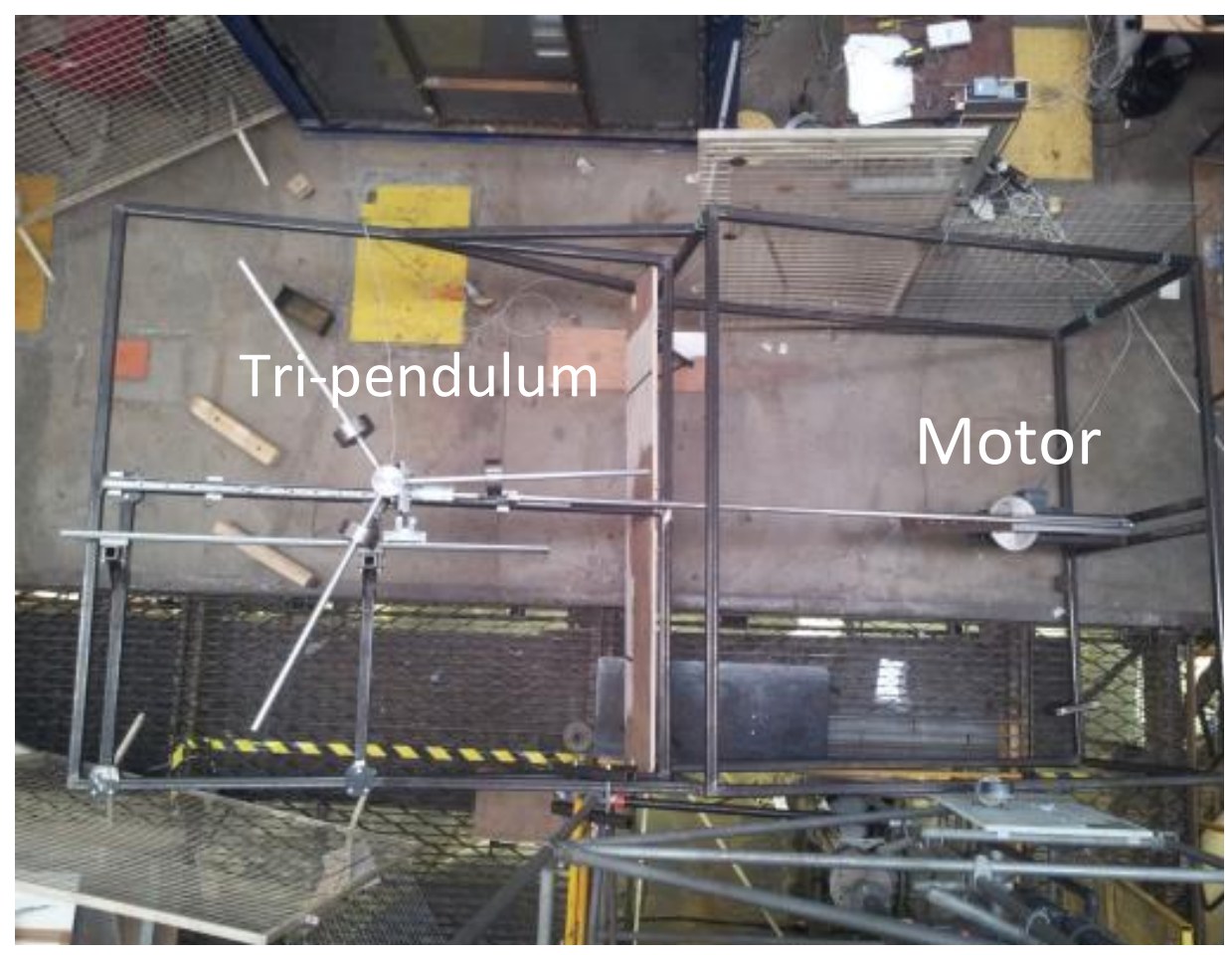

Figure 7: View of the full experimental rig.

The purpose of the experiments was to achieve rotational motion of the pendulum at low values of $\lambda$, which could not be reached at the vertical 


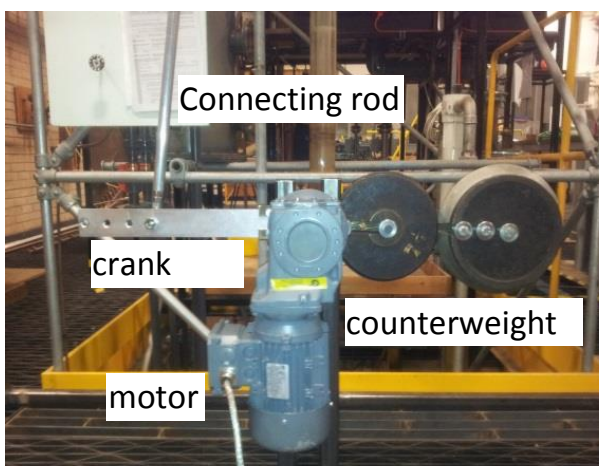

(a)

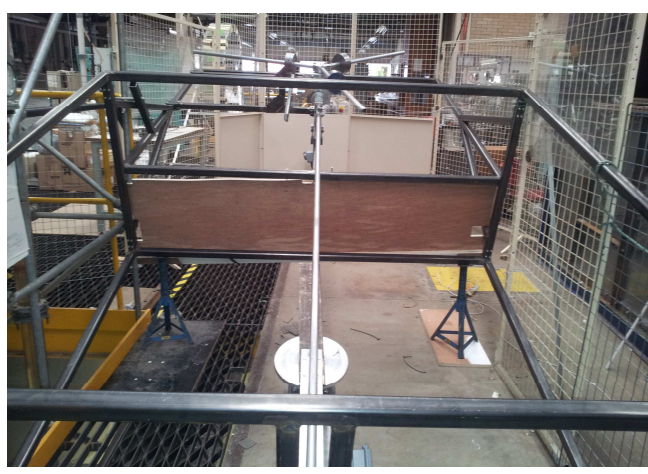

(b)

Figure 8: Parts of the apparatus; (a) The motorizing sub-system of the rig.; (b) View of the full experimental rig tilted from the horizontal level at a constant angle.

Table 1: Specifications of the experimentally recorded responses of the tri-pendulum to vertical excitation.

\begin{tabular}{llllllll}
\hline$\omega(\mathrm{rad} / \mathrm{s})$ & $L_{1}(\mathrm{~m})$ & $L_{2}(\mathrm{~m})$ & $\Omega(\mathrm{rd} / \mathrm{s})$ & $\nu$ & $\lambda$ & Zone & Resp \\
\hline 1.98 & 0.357 & 0.18 & 2.64 & 0.75 & 0.2 & Blue & $\mathrm{F}$ \\
1.98 & 0.476 & 0.25 & 2.33 & 0.85 & 0.2 & Red & $\mathrm{R}$ \\
2.426 & 0.476 & 0.25 & 2.33 & 0.85 & 0.3 & Green & $\mathrm{R} / \mathrm{O}(68 \%)$ \\
2.426 & 0.432 & 0.225 & 2.43 & 1.00 & 0.3 & Red & $\mathrm{R}$ \\
2.426 & 0.283 & 0.2 & 1.94 & 1.25 & 0.3 & Blue & $\mathrm{F}$ \\
\hline
\end{tabular}

motion of the suspension point. It has been reported in [33] that no rotational motion was observed for values of $\lambda<0.5$. First a set of free vibration tests was conducted to calculate the values of the viscous damping coefficient for different angles $\beta$. Using the logarithmic decrement procedure the equivalent viscous damping coefficient was found to be $\gamma=0.01$ for $\beta=15^{\circ}$. This value of the damping coefficient was used to produce the corresponding PSMs and identify points of interest for the experimental study, marked by white crosses in Figure 9. 


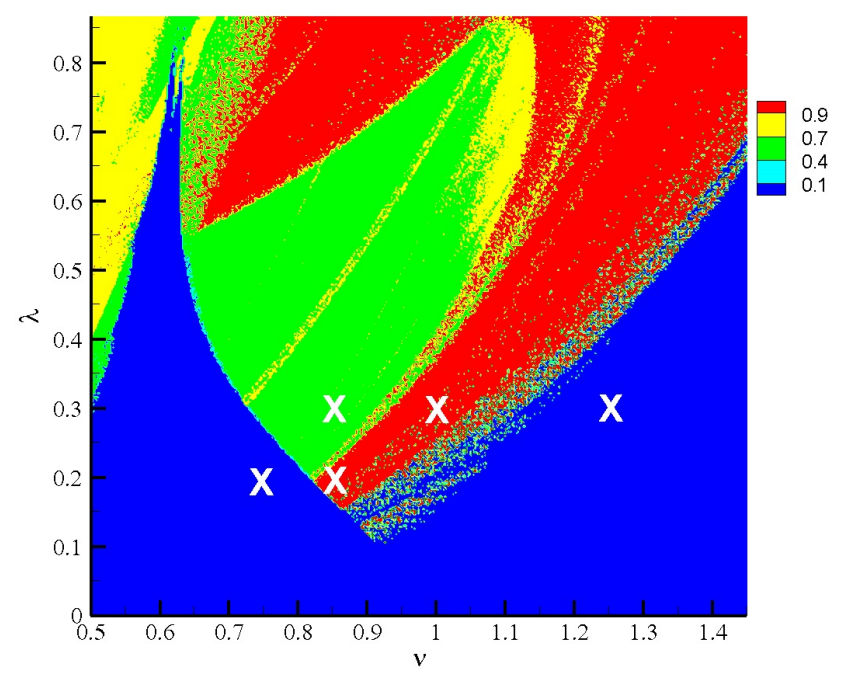

Figure 9: PSM for $\beta=15^{\circ}$. The experimental test points are marked by crosses

In Table 1 the results of the experimental tests are presented for $\beta=15^{\circ}$ and $A *=0.5 \mathrm{~m}$. As expected rotational $(R)$ motion has been observed in red domains, whereas no rotations or oscillations $(F)$ were observed in the blue regions. Indicatively, phase space trajectories are shown in 10 corresponding to $\nu=0.85, \lambda=0.2$, revealing the close but not ideal corroboration. Nevertheless, the qualitative characterization of the response still holds. In the green domain rotational and oscillatory $(R / O)$ motion has been observed, however high percentage of the observed rotational motion, close to the upper value in that range, was not expected and was obtained by averaging over a number of tests. It should be stressed that it is difficult to assure proper initial conditions from one experiment to another due to various uncertainties presented in the rig design, therefore basins of attraction were constructed numerically first for the test points. In complex cases the assessment of the attractor's robustness can be evaluated using the integrity concept [34], whereas in the present case such a technique was not useful 


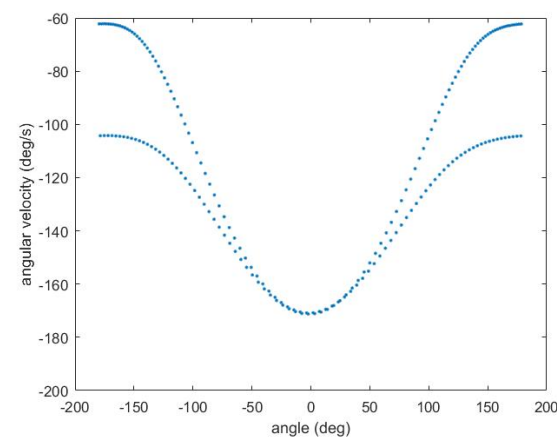

(a)

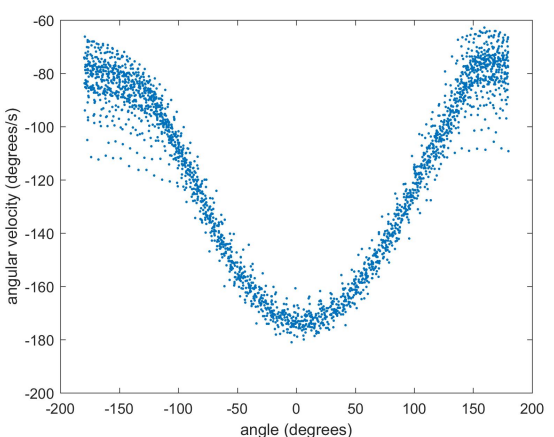

(b)

Figure 10: Phase space trajectories for $\beta=15^{\circ}$ and $\nu=0.85, \lambda=0.2$ coming (a) from numerical simulations (b) from experimental data;

due to simplicity of the plot, where almost each and every point led to the rotational motion, if the point $(\lambda, \nu)$ was in the red domain.

\section{Energy harvesting considerations}

The obtained numerical results have demonstrated the shift in the resonance frequency ratio from $\nu=2$ for $\beta=90^{\circ}$ to $\nu=0.7$ for $\beta=10^{\circ}$. It suggests that the original tri-pendulum design is not required and can be substituted by a regular pendulum. However, to harvest more energy and be able to control the the pendulum's natural frequency it is reasonable to add a flywheel to the design. Then the equation of motion will be identical to (5) with an additional inertia component:

$$
M l^{2}(1+n) \ddot{\theta}+b \dot{\theta}+M g l \sin \beta \sin \theta=M l \ddot{f}(t) \sin \theta, n=\frac{m R^{2}}{M l^{2}}
$$

where $m, R$ - the flywheel mass and radius, assuming the flywheel is a disk. This simple mechanism can help in reducing the natural frequency, increasing the power output and mitigate the pendulum motion through small fluctuations of the excitation. 
Considering now the power capture mechanism, let us consider that an inductive electromagnetic transducer is driven by the angular motion of the pendulum. In such a case, the electromotive force is the coupling between the mechanical system and the electric circuit as in

$$
\begin{aligned}
& M L^{2} \ddot{\theta}+b \dot{\theta}+M g l \sin \beta \sin \theta-M l \ddot{f}(t) \sin \theta-\Theta I=0, \\
& L_{c} \dot{I}+r_{w}+R_{L} I+\Theta \dot{\theta}=0
\end{aligned}
$$

where $I$ is the current flowing in the circuit, $\Theta$ is the coupling parameter that contains the radius of generator, which can be as big as the pendulum or buoy radius (6 meters for the full scale device). Furthermore, applying the same time non-dimensionalization as before and normalizing by the inertia:

$$
\begin{aligned}
& \theta^{\prime \prime}+\gamma \theta^{\prime}+[\sin \beta+\lambda \cos q(t)] \sin \theta-\frac{\Theta}{M l^{2} \Omega^{2}} I=0, \\
& L_{c} I^{\prime}+\frac{r_{w}+R_{L}}{\Omega} I+\Theta \theta^{\prime}=0,
\end{aligned}
$$

which corresponds to the deterministic case from Eq. (11). Furthermore, quite often, the circuit's inductance is much smaller than its resistance, i.e. $L_{c} \ll r_{w}+R_{L}$, which is also dominated by the load's resistance, $r_{w} \ll R_{L}$. This leads the second equation in (14) to give:

$$
I=-\frac{\Theta \Omega}{R_{L}} \theta^{\prime}
$$

Hence, the pendulum's equation of motion results in:

$$
\theta^{\prime \prime}+\left(\gamma+\gamma_{e}\right) \theta^{\prime}+[\sin \beta+\lambda \cos q(t)] \sin \theta=0
$$

where $\gamma_{e}=\Theta^{2} / R_{L} M l^{2} \Omega$. This shows that the electrical generator influences the energy harvesting of the device through the damping term. Considering now electrical power $P=I^{2} R_{L}$ and with the current expression in (15), the normalized power reads: 


$$
P_{n}=\frac{P}{M l^{2} \Omega^{3}}=\gamma_{e} \theta^{\prime 2}
$$

Note that the normalized power in Eq. (17) depends on the normalized angular velocity and thus it is not fixed. Therefore it is possible to plot the normalized power of the WEC (17) for a given value of the load (damping) and it is assume for the simplicity sake that $\gamma_{e}>>\gamma$. It should be mentioned that when the damping changes the PSM maps will be modified, leading to a new set of optimal values $(\nu, \lambda)$, which are inside the red domains. Figure 11 shows three curves of normalized power $P_{n}(W)$ corresponding to different sets of $(\nu, \lambda)$ as a function of $\beta$. The orange line corresponds to the point located in the low red part of the PSM map $\left(\nu_{1}, \lambda_{1}\right)$, the green line corresponds to the point located above the previous one but in the green area of PSM $\left(\nu_{1}, \lambda_{2}\right)$, whereas the red line corresponds to the point located to the right from the green one but again inside the red area of PSM, so that $\left(\nu_{2}, \lambda_{2}\right)$, where $\lambda_{2}>\lambda_{1}$ and $\nu_{2}>\nu_{1}$. To be more specific for $\beta=10^{\circ}$ the following set values of the

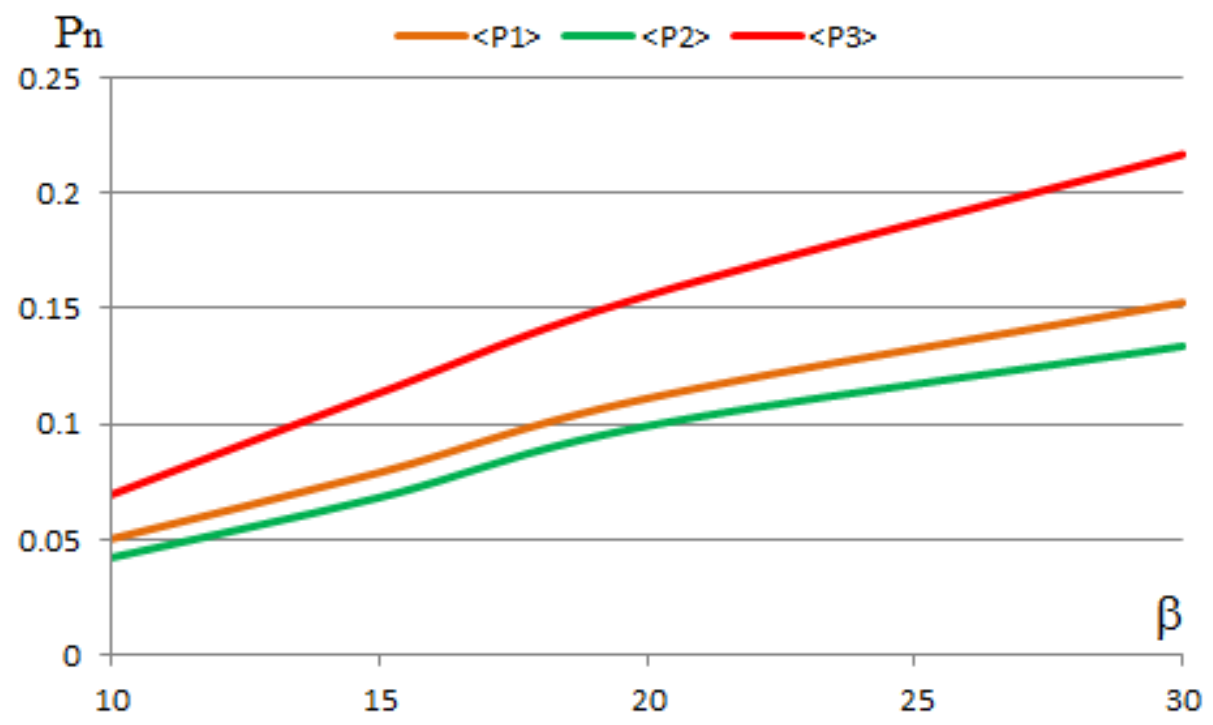

Figure 11: Normalized averaged electrical power (17) for different set of $(\nu, \lambda)$. 
PSM has been used for figure 11: $\nu_{1}=0.7, \lambda_{1}=0.2, \nu_{2}=0.8, \lambda_{2}=0.3$, and this trend of selecting values of $(\nu, \lambda)$ was kept for other values of $\beta$. It can be seen, as expected that the amount of harvested power increases when one moves up and to the right along the corresponding PSMs, staying inside the red domain. In the green domain the percentage of rotational motion is lower than that in the red domain, therefore the averaged energy is lower and as a result the power delivered is lower too (green curve in Figure 11). In the absolute values the harvested power of the device of 6 meters long, $1000 \mathrm{~kg}$ mass and $\gamma_{e}=0.1, \Omega=1$ will vary from $2.5 \mathrm{~kW}$ for $\beta=10^{\circ}$ to $7.8 \mathrm{~kW}$ for $\beta=30^{\circ}$. However this number does not include the fact that the electrical generator can also be as big as the buoy radius, which increases the relative speed thereby increasing the harnessed power and the load will apparently be also at least orders of magnitude bigger than one presented in the figure above.

\section{Conclusion}

The paper presents a novel WEC concept based on the parametrically excited pendulum. The initially proposed point absorber concept, driven by the heaving motion of waves, proved to be unfeasible due to low amplitude and frequency of waves in a regular sea state. The developed new design, presented in this paper, has offered a new arrangement, which allows the parametric pendulum achieving the rotational response at a regular sea state. The new low gravity concept uses both the heaving and surging motion of waves transforming it directly to the rotational motion through the parametrically excited pendulum. The dynamics of the novel device has been numerically and experimentally investigated and the results have been presented in this paper. The results have confirmed the increase in the rota- 
tional potential of the pendulum by moving the dominant rotational domains to significantly lower values of $\lambda$. Since the device is meant to harvest energy, the secondary goal was the development of the device, which would work in a regular sea state without any permanent active control interaction. Although this goal has been achieved and the paper has not discussed any control strategies related to the pendulum motion, the proposed design offers potentially versatile control over the device through the ramp angle $\beta$, the pendulum moment of inertia and the buoy buoyancy. The paper considered a coupling of the pendulum with a simple electromagnetic generator and provides some results on power harnessed by the device. It should be stressed that this paper provided the mechanical design of the device and has not been focused on the hydrodynamic properties of the buoy (its shape, buoyancy), which should be a part of another complex study.

\section{Acknowledgment}

The experimental study, presented in this paper, would not have been possible without support of the technicians from the mechanical workshop of Heriot-Watt University. The authors would particularly like to express their gratitude to Mr. Richard Kinsella and Mr. Ian Harrower.

\section{References}

[1] A. F. d. O. Falcão, Wave energy utilization: A review of the technologies, Renewable and Sutainable Energy Review 14 (2010) 899-918.

[2] Carbon Trust, Accelerating marine energy - The potential for cost reduction, Tech. Rep., Carbon Trust, 2011. 
[3] M. Wiercigroch, A. Najdecka, V. Vazirii, Nonlinear Dynamics of Pendulums System for Energy Harvesting, in: Vibration problems ICOVP 2009, Springer, 35-42, 2009.

[4] X. Xu, M. Wiercigroch, M. P. Cartmell, Rotating orbits of a parametrically-excited pendulum, Chaos Soliton Fractal 23 (2005) 15371548.

[5] B. Horton, S. Lenci, E. Pavlovskaia, F. Romeo, G. Rega, M. Wiercigroch, Stability Boundaries of Period-1 Rotation for a Pendulum Under Combined Vertical and Horizontal Excitation, J. Applied Nonlin. Dyn. 2 (2) (2013) 103-126.

[6] R. H. Avanço, H. A. Navarro, R. M. L. R. F. Brasil, J. M. Balthazar, Á. M. Bueno, A. M. Tusset, Statements on nonlinear dynamics behavior of a pendulum, excited by a crank-shaft-slider mechanism, Meccanica 51 (6) (2016) 1301-1320.

[7] A. Tusset, F. Janzen, V. Piccirillo, R. Rocha, J. Balthazar, G. Litak, On nonlinear dynamics of a parametrically excited pendulum using both active control and passive rotational (MR) damper, Journal of Vibration and Control (2017) 1-13.

[8] B. Horton, J. Sieber, J. M. T. Thompson, M. Wiercigroch, Dynamics of the Nearly Parametric Pendulum, Int J Non-linear Mech 46 (2011) 436-442.

[9] P. Brzeski, P. Perlikowski, S. Yanchuk, T. Kapitaniak, The dynamics of the pendulum suspended on the forced Duffing oscillator, J Sound Vib $331(24)(2012) 5347-5357$. 
[10] C. Sartorelli, W. Lacarbonara, Parametric resonances in a base-excited double pendulum, Nonlinear Dyn 69 (2012) 1679-1692.

[11] J. Strzalko, J. Grabski, J. Wojewoda, M. Wiercigroch, T. Kapitaniak, Synchronous rotation of the set of double pendula: Experimental observations, Chaos 22 (4) (2012) 047503.

[12] D. Yurchenko, P. Alevras, Dynamics of the N-pendulum and its application to a wave energy converter concept, Int. J.l of Dynamics and Control 1 (4) (2013) 290-299.

[13] M. Kapitaniak, K. Czolczynski, P. Perlikowski, A. Stefanski, T. Kapitaniak, Synchronous states of slowly rotating pendula, Physics Reports doi:10.1016/j.physrep.2014.02.008.

[14] T. Andreeva, P. Alevras, D. Naess, Arvid and, Dynamics of a parametric rotating pendulum under a realistic wave profile, Int. J. of Dynamics and Control .

[15] X. Xu, E. Pavlovskaia, M. Wiercigroch, F. Romeo, S. Lenci, Dynamic interactions between parametric pendulum and electro-dynamical shaker, ZAMM 87 (2007) 172-186.

[16] S. Lenci, M. Brocchini, C. Lorenzoni, Experimental rotations of a pendulum on water waves, J Comput Nonlinear Dyn 7 (1) (2012) 011007.

[17] S. Lenci, G. Rega, Experimental versus theoretical robustness of rotating solutions in a parametrically excited pendulum: a dynamical integrity perspective, Physica D: Nonlinear Phenomena 240 (9) (2011) 814-824.

[18] S. Lenci, On the Production Of Energy From Sea Waves By a Rotating 
Pendulum: A Preliminary Experimental Study, J Applied Nonlin Dyn 3 (2) (2014) 187-201.

[19] S.-H. Teh, K.-C. Woo, H. Demrdash, Rotating orbits of pendulum in stochastic excitation, Journal of Theoretical and Applied Mechanics $54(3)$.

[20] F. E. Dotti, F. Reguera, S. P. Machado, Damping in a parametric pendulum with a view on energy harvesting, Mechanics Research Communications 81 (2017) $11-16$.

[21] M. Marszal, B. Witkowski, K. Jankowski, P. Perlikowski, T. Kapitaniak, Energy harvesting from pendulum oscillations, International Journal of Non-Linear Mechanics (2017) -.

[22] D. Yurchenko, A. Naess, P. Alevras, Pendulum's rotational motion governed by a stochastic Mathieu equation, Probabilist Eng Mech 31 (2013) $12-18$.

[23] P. Alevras, D. Yurchenko, Stochastic rotational response of a parametric pendulum coupled with an SDOF system, Probabilist Eng Mech 37 (2014) 124-131.

[24] P. Alevras, D. Yurchenko, A. Naess, Stochastic synchronization of rotating parametric pendulums, Meccanica 49 (8) (2014) 1945-1954.

[25] P. Alevras, D. Yurchenko, R. Bobryk, Stability of an autoparametic pendulum with impacts, J Sound Vib 33 (2014) 7233 - 7247.

[26] A. Najdecka, S. Narayanan, M. Wiercigroch, Rotary motion of the parametric and planar pendulum under stochastic wave excitation, International Journal of Non-Linear Mechanics 71 (2015) 30 - 38. 
[27] A. H. Nayfeh, D. T. Mook, Nonlinear Oscillations, Wiley, New York, 1995.

[28] A. J. Nambiar, D. I. Forehand, M. M. Kramer, R. H. Hansen, D. M. Ingram, Effects of hydrodynamic interactions and control within a point absorber array on electrical output, International Journal of Marine Energy 9 (2015) $20-40$.

[29] R. Gomesa, M. Lopesb, J. Henriquesa, L. Gatoa, A.Falcão, The dynamics and power extraction of bottom-hinged plate wave energy converters in regular and irregular waves, Ocean Engineering 96 (2015) 86-99.

[30] D. Iourtchenko, Response spectral density of linear systems with external and parametric non-Gaussian, delta-correlated excitation, Probabilistic Engineering Mechanics 18 (1) (2003) 31 - 36.

[31] D. Yurchenko, P. Alevras, Stochastic dynamics of a parametrically base excited rotating pendulum, Procedia IUTAM 6 (2013) 160-168.

[32] W. Chai, A. Naess, B. J. Leira, Filter models for prediction of stochastic ship roll response, Probabilistic Engineering Mechanics 41 (2015) 104 114.

[33] P. Alevras, I. Brown, D. Yurchenko, Experimental investigation of a rotating parametric pendulum, Nonlin Dyn 81 (1) (2015) 201 -213.

[34] S. Lenci, G. Rega, Experimental versus theoretical robustness of rotating solutions in a parametrically excited pendulum: A dynamical integrity perspective, Physica D: Nonlinear Phenomena 240 (9-10) (2011) 814824. 